\title{
RESEARCH
}

Open Access

\section{Human amnion-derived mesenchymal stem cells promote osteogenic differentiation of lipopolysaccharide-induced human bone marrow mesenchymal stem cells via ANRIL/ miR-125a/APC axis}

Yuli Wang ${ }^{1,2}$, Fengyi LV ${ }^{1}$, Lintong Huang ${ }^{1}$, Hengwei Zhang ${ }^{3}$, Bing Li ${ }^{1,2}$, Weina Zhou ${ }^{1,4}$, Xuan Li ${ }^{1}$, Yifei Du ${ }^{1,2^{*}}$, Yongchu Pan ${ }^{1,5^{*}}$ and Ruixia Wang ${ }^{1,6^{*}}$

\begin{abstract}
Background and aim: Periodontitis is a chronic inflammatory disease inducing the absorption of alveolar bone and leading to tooth loss. Human amnion-derived mesenchymal stem cells (HAMSCs) have been used for studying inflammatory processes. This study aimed to explore the role of long noncoding RNA (IncRNA) antisense noncoding RNA in the INK4 locus (ANRIL) in HAMSC-driven osteogenesis in lipopolysaccharide (LPS)-induced human bone marrow mesenchymal stem cells (HBMSCs).

Methods: The cells were incubated with a co-culture system. Reactive oxygen species (ROS) level and superoxide dismutase (SOD) activity were used to detect the oxidative stress level. Flow cytometry was performed to determine cell proliferation. The alkaline phosphatase (ALP) activity, Alizarin red assay, cell transfection, and rat mandibular defect model were used to evaluate the osteogenic differentiation. Quantitative real-time reverse transcription-polymerase chain reaction (RT-PCR), Western blot analysis, dual-luciferase reporter assay, and immunofluorescence staining were used to evaluate the molecular mechanisms.

Results: This study showed that HAMSCs promoted the osteogenesis of LPS-induced HBMSCs, while the ANRIL level in HBMSCs decreased during co-culture. ANRIL had no significant influence on the proliferation of LPS-induced HBMSCs. However, its overexpression inhibited the HAMSC-driven osteogenesis in vivo and in vitro, whereas its knockdown reversed these effects. Mechanistically, this study found that downregulating ANRIL led to the overexpression of microRNA-125a (miR-125a), and further contributed to the competitive binding of miR-125a and adenomatous polyposis coli (APC), thus significantly activating the Wnt/ $\beta$-catenin pathway.

(Continued on next page)
\end{abstract}

\footnotetext{
*Correspondence: duyifeinjmu@163.com; panyongchu@njmu.edu.cn;

wangruixianjmu@163.com

'Jiangsu Key Laboratory of Oral Diseases, Nanjing Medical University, Nanjing,

China

Full list of author information is available at the end of the article
}

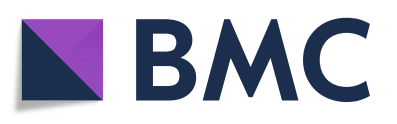

(- The Author(s). 2021 Open Access This article is licensed under a Creative Commons Attribution 4.0 International License, which permits use, sharing, adaptation, distribution and reproduction in any medium or format, as long as you give appropriate credit to the original author(s) and the source, provide a link to the Creative Commons licence, and indicate if changes were made. The images or other third party material in this article are included in the article's Creative Commons licence, unless indicated otherwise in a credit line to the material. If material is not included in the article's Creative Commons licence and your intended use is not permitted by statutory regulation or exceeds the permitted use, you will need to obtain permission directly from the copyright holder. To view a copy of this licence, visit http://creativecommons.org/licenses/by/4.0/. The Creative Commons Public Domain Dedication waiver (http://creativecommons.org/publicdomain/zero/1.0/) applies to the data made available in this article, unless otherwise stated in a credit line to the data. 
(Continued from previous page)

Conclusion: The study indicated that HAMSCs promoted the osteogenic differentiation of LPS-induced HBMSCS via the ANRIL/miR-125a/APC axis, and offered a novel approach for periodontitis therapy.

Keywords: Adenomatous polyposis coli, $\beta$-Catenin, Human amnion-derived mesenchymal stem cells, Human bone marrow mesenchymal stem cells, Lipopolysaccharide, Long noncoding RNA ANRIL, microRNA-125a, Osteogenic differentiation

\section{Introduction}

Periodontitis is a group of plaque-induced chronic inflammatory processes characterized by alveolar bone deficiency and tooth loss. So far, strategies for healing periodontitis are challenging; they may have remarkable individual differences and increase patients' burden. Lipopolysaccharide (LPS), the major virulence factor of the outer membranes of Gram-negative bacteria, is related to the immune responses of periodontitis $[1,2]$. Human bone marrow mesenchymal stem cells (HBMSCs) are commonly used in alveolar bone regeneration due to their self-renewal capacity and availability [3]. However, the LPS-induced pathological status is detrimental to HBMSC retention and survival. Human amnion-derived mesenchymal stem cells (HAMSCs), harvested in a noninvasive manner, have superior immunomodulatory properties and fewer ethical concerns [4]. A previous report showed that HAMSCs modulated the osteogenic differentiation and alleviated oxidative stress in LPS-induced HBMSCs [5]. Many studies explored the osteogenesis of HBMSCs against LPS by HAMSCs; however, the curative effect and regulatory mechanism still need to be fully investigated.

Long noncoding RNAs (lncRNAs) is a critical subgroup of noncoding RNAs (ncRNAs) more than $200 \mathrm{nu}$ cleotides in length [6]; they are involved in a broad spectrum of biological control and pathology [7, 8]. Antisense noncoding RNA in the INK4 locus (ANRIL) is an IncRNA first found in melanoma and neural system tumor [9]. Recent studies indicated the relationship between ANRIL and periodontitis [10, 11]. Higher level of ANRIL was found in Porphyromonas gingivalis-induced periodontal tissue [12]. This study investigated whether HAMSCs promoted the osteogenesis of LPS-induced HBMSCs via ANRIL.

MicroRNAs (miRNAs) are a class of small noncoding RNAs that activate the expression of protein-encoding genes by binding to the 3'-UTR of the target mRNAs [13]. Recent studies demonstrated that ANRIL participated in the malignant transformation and progression of various diseases by acting as a sponge of microRNA125a (miR-125a) $[14,15]$. However, whether the ANRIL/ miR-125a axis is involved in the HAMSC-driven osteogenesis remains undetermined. This study investigated the effect of ANRIL and its underlying mechanism.
HAMSCs promoted osteogenic differentiation in LPSinduced HBMSCs, while ANRIL had the opposite effect. Moreover, downregulating ANRIL induced miR-125a overexpression, further inhibiting adenomatous polyposis coli (APC) and activating Wnt/ $/$-catenin signaling. These new references for the lncRNA-miRNA-mRNA functional network may be a powerful strategy for the therapy of periodontitis.

\section{Materials and methods \\ Cell culture and co-culture system}

All experiments were approved by the ethics and research committee of Nanjing Medical University (Permit Number: 2018-190). Informed consent was obtained from all the participants. HAMSCs were obtained from the discarded amniotic membrane, and HBMSCs were collected from patients undergoing sagittal split ramus osteotomy (SSRO). The cells were isolated and maintained as reported $[16,17]$. The cells in three to five passages were used in this study. An HAMSCs/HBMSCs Transwell co-culture system was established as previously described [5]. HBMSCs were seeded at an initial cell density of $5 \times 10^{4}$ cells $/ \mathrm{cm}^{2}$ in six-well culture plates. Transwells were placed in other six-well culture plates, and HAMSCs were seeded at the same ratios $\left(5 \times 10^{4}\right.$ cells/Transwell). Following the attachment of the cells (approximately $24 \mathrm{~h}$ ), HBMSCs were subjected to a 24-h treatment with serum-free medium or Escherichia coli LPS $(1 \mu \mathrm{g} / \mathrm{mL})$ to induce inflammatory responses. After washing with phosphate-buffered saline, Transwells containing HAMSCs were transferred to the corresponding wells of the six-well culture plate containing HBMSCs.

\section{Quantitative real-time reverse transcription-polymerase chain reaction ( $R T-P C R)$}

RNA isolation and cDNA transcription were performed using TRIzol reagent (Invitrogen, NY, USA) and reverse transcription kit (Applied Biosystems, CA, USA). RTPCR was conducted as previously reported [18]. The primer sequences used are listed in Table 1. Human glyceraldehyde-3-phosphate dehydrogenase was used as a reference for estimating the levels of lncRNAs and mRNAs, whereas human U6 was used to normalize miRNAs. The fold changes in gene expression were determined by the $2^{-\Delta \Delta \mathrm{Ct}}$ method. 
Table 1 Primers used for quantitative real-time reverse transcription polymerase chain reaction

\begin{tabular}{lll}
\hline Genes & Sense primer(5'-3') & Anti-sense primer(5'-3') \\
\hline ALP & AGAACCCAAAGGCTTCTC & CTTGGCTTTCCTTCATGGT \\
RUNX2 & TCTTAGAACAAATCTGCCCTT & TGCTTTGGTCTTGAAATCACA \\
& T & \\
OCN & AGCAAAGGTGCAGCCTTGT & GCGCCTGGGTCTCTTCACT \\
OSX & CCTCCTCAGCTCACCTTCTC & GTTGGAGCCCAAATAGAAA \\
ANRIL & CCCTAGCTACATCCGTCA & CCACAGCTACATATGCGT \\
& CCTGA & TTACA \\
APC & AAAGTGAGCAGCTACCACG & CCTGGAGTGATCTGTTAGTCG \\
- & AGCTGACAACTTTCACACC & AATGGGGATGTTATCTTC \\
Catenin & & \\
\hline
\end{tabular}

Reactive oxygen species (ROS) level and superoxide dismutase (SOD) activity

Flow cytometry was used to determine LPS-induced ROS by measuring the intensity of $2^{\prime}, 7^{\prime}$-dichlorofluorescin fluorescence after $48 \mathrm{~h}$ as previously reported [5]. The SOD activity was detected using a xanthine oxidase assay kit (Jiancheng Corp., Nanjing, China) following the manufacturer's protocols [19].

\section{Cell transfection}

Recombinant lentiviruses containing full-length ANRI L, scramble control (NC), targeting ANRIL, and scramble control (shNC) were obtained from GenePharma Company (Shanghai, China). Those lentiviruses were named Lenti-ANRIL, Lenti-NC, Lenti-sh ANRIL, and Lenti-shNC, respectively. HBMSCs transfected with miRNA plasmids (RiboBio, Guangzhou, China) were prepared using transfection reagent riboFECTTM CP (RiboBio). The mutated binding sites of miR-125a in luciferase reporter vectors containing APC were constructed by site-directed mutagenesis.

\section{Cell proliferation assay}

HBMSCs were collected after 1, 3, and 5 days. Flow cytometry (BD Biosciences, NJ, USA) was performed to determine the cell viability as previously reported [20]. G0, G1, S, and G2 M phases were determined using MODFIT LT 3.2 (Verity Software House, ME, USA).

\section{Alkaline phosphatase (ALP) and alizarin red assay}

After 7 days of osteogenic induction, ALP staining was used to detect the activity with an NBT/BCIP staining kit (CoWin Biotech, Beijing, China) and an ALP assay kit (Jiancheng Corp, Nanjing, China) as previously reported [21, 22]. Mineralized matrix formation was determined after 14days of osteogenic induction as previously reported [23].

\section{Western blot analysis}

Western blot analysis was performed as previously reported [24]. The primary antibodies were as follows: anti-ALP (ab83259) (1:1000), anti-osteocalcin (OCN) (ab133612) (1:1000), and anti-Osterix (OSX) (ab209484) (1:1000) (all from Abcam, MA, USA; and RUNX2 (D1L7F) rabbit mAb \#12556 (1:1000), APC antibody \#2504 (1:1000), -catenin (D10A8) XP Rabbit mAb \#8480 (1:1000), and -actin (8H10D10) mouse mAb \#3700 (1:1000) (all from Cell Signaling Technology MA, USA). -Actin served as an internal control. Relative densitometry analysis of the Western blot was carried out using ImageJ software. Relative protein levels were quantified as the ratio of the level of target protein to the level of -actin, in each group.

\section{In vivo bone formation assay}

Approximately $10^{5}$ cells $\left(5 \times 10^{4}\right.$ HAMSCs and $5 \times 10^{4}$ $\mathrm{HBMSCs}^{\mathrm{NC}} / \mathrm{HBMSC}{ }^{\text {ANRIL }} / \mathrm{HBMSCs}{ }^{\text {shNC }} / \mathrm{HBMSC}{ }^{\text {shAN- }}$ RIL pretreated with LPS) were attached to each HA/TCP biomaterial $(\Phi 5 \times \mathrm{H} 2 \mathrm{~mm}$, Sichuan University, Chengdu, Sichuan, China). After $12 \mathrm{~h}$, the complexes were subcutaneously implanted into the rat mandibular defect area designed as previously reported (four female nude rats per group, with an average weight of $280 \mathrm{~g}$ ) [23]. All animal experiments were conducted in compliance with the regulations and guidelines of the Nanjing Medical University institutional animal care.

\section{Micro-computed tomography (micro-CT) analysis}

Mandibles were harvested for micro-CT analysis after 8 weeks of implantation as previously reported [25]. Bone volume ratio $(\mathrm{BV} / \mathrm{TV}, \%)$ was calculated.

\section{Histological observation}

Mandible samples were harvested and analyzed using hematoxylin and eosin $(\mathrm{H} \& \mathrm{E})$, Masson trichrome, and immunohistochemical staining. Primary antibodies against RUNX2 (1:300 dilution) were used for immunohistochemical analysis as previously reported [23]. Positive areas were observed under the microscope.

\section{Dual-luciferase reporter assay}

Luciferase assays were performed using the Lipofectamine 2000 and Dual-Luciferase Reporter Assay System as previously reported [26].

\section{Immunofluorescence staining}

Primary antibody [ -Catenin (D10A8) XP Rabbit mAb \#8480 (1:100), Cell Signaling Technology], and DAPI were used to perform immunofluorescence staining as previously reported [23]. Images were captured under an inverted fluorescence microscope (Olympus, Japan). 


\section{Statistical analysis}

The data were expressed as the mean and standard deviation of at least three independent samples. Comparisons between two groups were analyzed using the twotailed, unpaired Student's $t$ test. Comparisons among $\geq 3$ groups were performed using one-way analysis of variance followed by Tukey's multiple comparisons. $P<0.05$ indicated a statistically significant difference.

\section{Results}

LncRNA-ANRIL expression in LPS-induced HBMSCs decreased with HAMSC co-culture

A Transwell co-culture model was established, and related markers were detected to verify the previous finding that HAMSCs reduced oxidative stress and promoted osteogenic differentiation in LPS-induced HBMSCs. HAMSCs increased the expression of osteogenic markers, including ALP, RUNX2, and OCN (Fig. 1a). In addition, HAMSCs also decreased the LPS-induced oxidative stress level in HBMSCs, which was confirmed by ROS and SOD measurements (Fig. 1b, c). Meanwhile, RT-PCR showed that the levels of ANRIL in LPS-induced HBMSCs significantly decreased in a time-dependent manner with the HAMSC co-culture (Fig. 1d).

\section{LncRNA-ANRIL had no effects on LPS-induced proliferation of HBMSCs}

Then, stably expressing cells (HBMSCs ${ }^{\mathrm{NC}}$, HBMSCs ${ }^{\text {ANRI }}$ L, HBMSCs ${ }^{\text {shNC }}$, and HBMSCs ${ }^{\text {shANRIL }}$ ) were sorted and co-cultured with HAMSCs after LPS pretreatment. The cells were assigned to the following groups: HBMSCs; LPS: LPS + HBMSCs; HAMSCs: HAMSCs + LPS + HBMSCs; NC: HAMSCs + LPS + HBMSCs ${ }^{\mathrm{NC}}$; ANRIL: HAMSCs + LPS + HBMSCs ${ }^{\text {ANRIL }}$; shNC: HAMSCs + LPS + HBMSCs $^{\text {shNC }}$; shANRIL: HAMSCs + LPS +
HBMSCs ${ }^{\text {shANRIL }}$. The proliferation assay conducted using flow cytometry suggested that HAMSCs promoted the growth of LPS-induced HBMSCs, while no significant difference in S-phase checkpoints was detected among the HAMSC, NC, ANRIL, shNC, and shANRIL groups (Fig. 1e).

\section{HAMSCs promoted the osteogenesis of LPS-induced HBMSCs via downregulating LncRNA-ANRIL}

The osteogenic differentiation in HBMSCs is vital in reversing alveolar bone deficiency. Therefore, the present study investigated whether ANRIL participated in HAMSC-driven osteogenesis. HAMSCs enhanced ALP staining and activity compared with those in LPS groups, whereas ANRIL overexpression in HBMSCs inhibited the effect, and ANRIL knockdown promoted it (Fig. 2a). Besides, Alizarin red staining and quantification showed decreased matrix mineralization in the ANRIL group compared with the HAMSCs and NC groups, whereas ANRIL knockdown exhibited the opposite effects (Fig. 2b). RT-PCR and Western blot analysis showed that the mRNA and protein levels of ALP and RUNX2 (early-stage osteogenic markers) and OCN and OSX (late-stage markers) were decreased by ANRIL overexpression and increased by ANRIL knockdown (Fig. 2c, d).

Followed the in vitro study, this study examined the effect of ANRIL in vivo using the mandibular defect model (Fig. 3a). The percentage of mineralized volume fraction was measured. ANRIL decreased bone volume/ total volume (BV/TV) compared with the NC group, while increased BV/TV was detected in the shANRIL group compared with shNC group (Fig. 3b). H\&E and Masson staining showed less organized bone matrix and more fibrous tissue in the ANRIL group, while the shANRIL group exhibited better bone formation. The

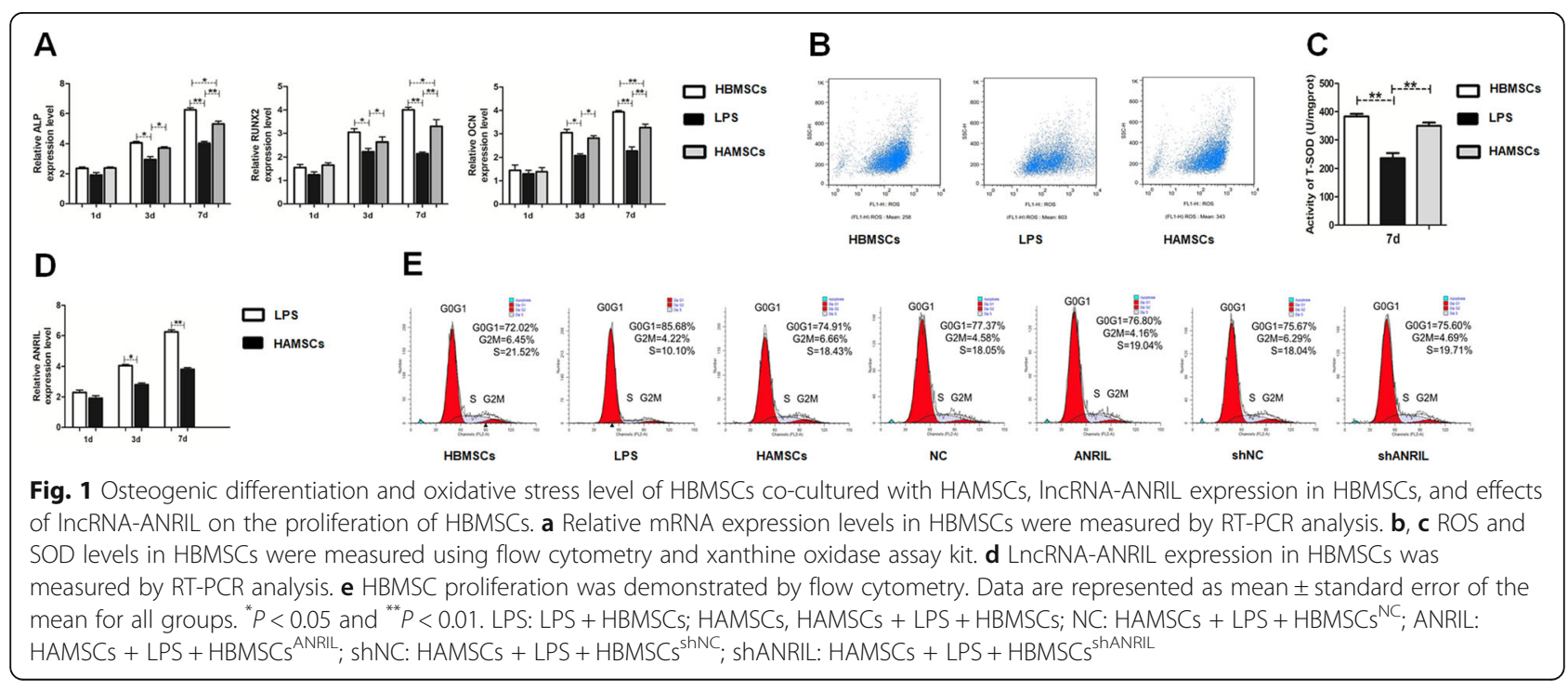




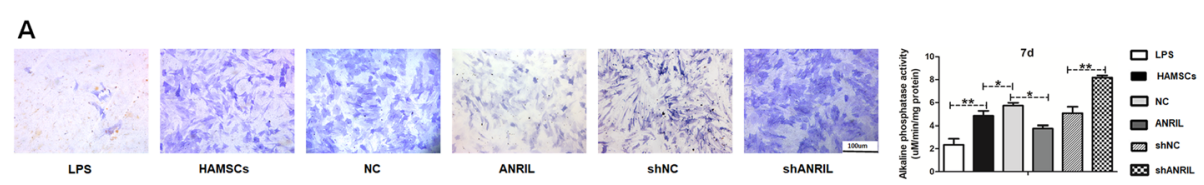

B

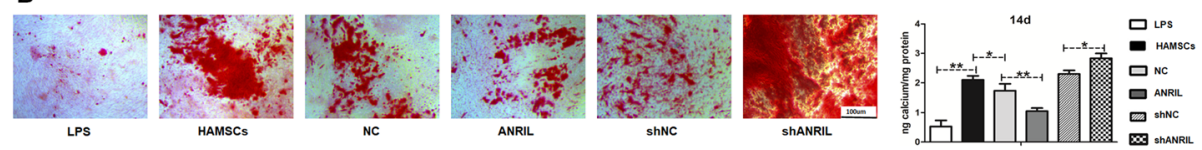

C

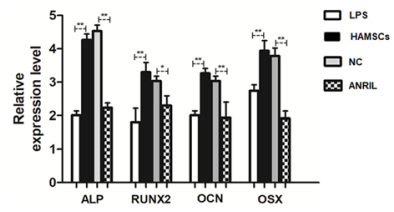

D
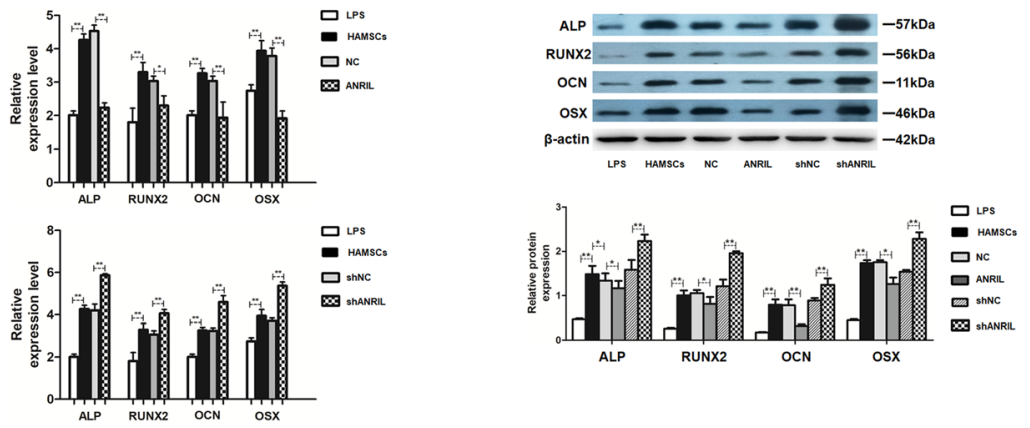

Fig. 2 HAMSCs promoted the osteogenesis of LPS-induced HBMSCs via the downregulation LnCRNA-ANRIL. a ALP staining and activity in HBMSCs. Scale bar, $100 \mu \mathrm{m}$. b Alizarin red staining and quantification in HBMSCs. Scale bar, $100 \mu \mathrm{m}$. c Relative mRNA expression levels in HBMSCs were measured by RT-PCR analysis. $\mathbf{d}$ Relative protein levels in HBMSCs were assessed by Western blot analysis. Data are represented as mean \pm standard error of mean for all groups. ${ }^{*} P<0.05$ and ${ }^{* *} P<0.01$. LPS: LPS + HBMSCs; HAMSCs: HAMSCs + LPS + HBMSCs; NC: HAMSCs + LPS + HBMSCS ${ }^{\text {NC }}$; ANRIL: HAMSCs + LPS + HBMSCs ${ }^{\text {ANRIL; }}$ shNC: HAMSCs + LPS + HBMSCs ${ }^{\text {shNC; }}$ shANRIL: HAMSCs + LPS + HBMSCs ${ }^{\text {ShANRIL }}$

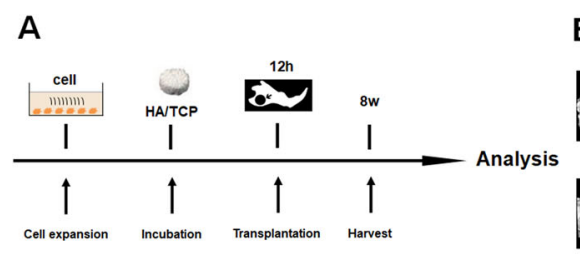

\section{B}

C
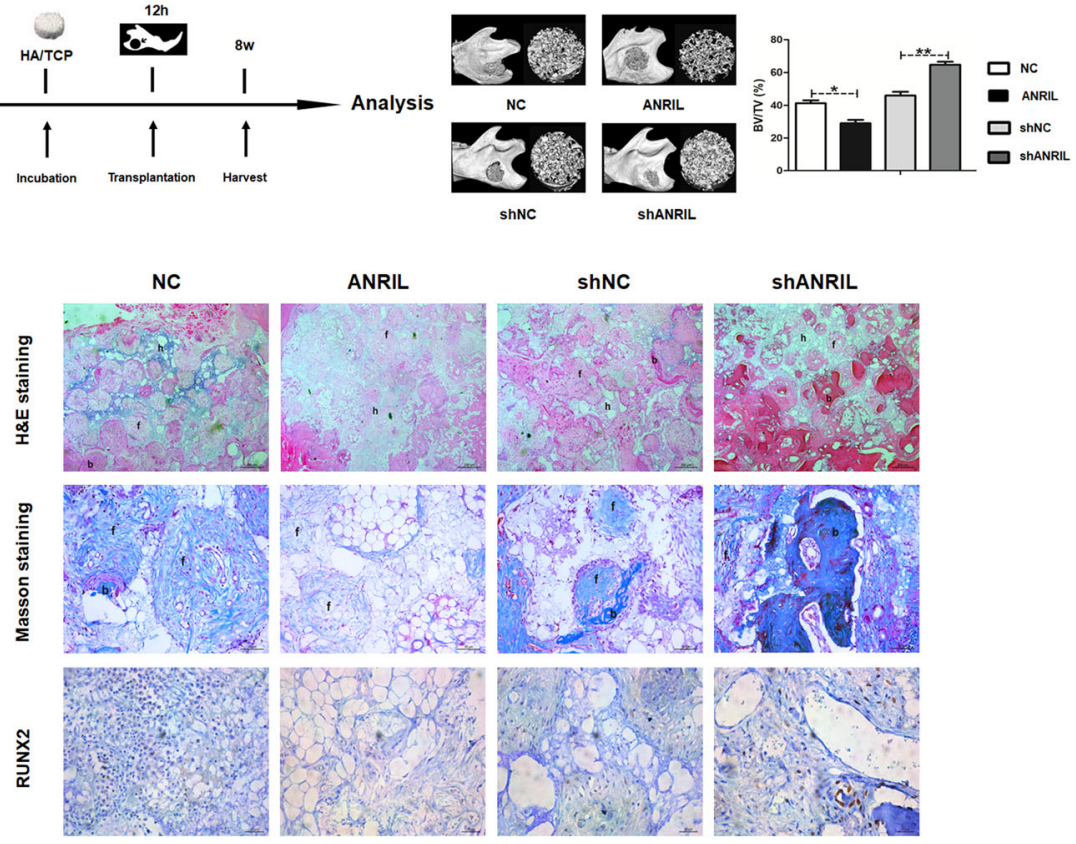

Fig. 3 LnCRNA-ANRIL in HBMSCs inhibited osteogenesis in vivo. a The cells in the NC, ANRIL, shNC, and shANRIL groups were transplanted subcutaneously into a rat critical-sized mandibular defect model. b Reconstructed 3D micro-CT images of the tissue-engineered bone and values of BV/TV. c H\&E staining, Masson staining, and immunohistochemical staining of RUNX2 in each group (b: bone-like tissues; h: HA/TCP scaffold; f: fibrous). Scale bar, $200 \mu \mathrm{m}$. Data are represented as mean \pm standard error of the mean for both groups. ${ }^{*} P<0.05$ and ${ }^{* *} P<0.01$. NC: HAMSCS + LPS + HBMSCs ${ }^{N C}$; ANRIL: HAMSCs + LPS + HBMSCs ${ }^{\text {ANRIL. }}$; shNC: HAMSCs + LPS + HBMSCs ${ }^{\text {shNC; }}$; ShANRIL: HAMSCs + LPS + HBMSCs ${ }^{\text {shANRIL }}$ 
immunohistochemical analysis also confirmed that RUNX2 expression was downregulated in the ANRIL group compared with the $\mathrm{NC}$ group and upregulated in the shANRIL group compared with the shNC group (Fig. 3c).

\section{MiR-125a negatively correlated with LncRNA-ANRIL}

This study next investigated the possible interaction between ANRIL and miR-125a. The putative miR-125abinding sequence of ANRIL was predicated using TargetScan software (Fig. 4a). The correlation between ANRIL and miR-125a was further examined using a dual-luciferase reporter assay to identify whether ANRIL could serve as an miRNA sponge to directly negatively regulate the expression of miR-125a. The results showed that miR-125a significantly attenuated the luciferase activity of ANRIL-WT, while no change was detected in ANRIL-Mut groups (Fig. 4b). The miR-125a expression was downregulated by ANRIL overexpression and upregulated by ANRIL knockdown (Fig. 4c). Further, miR125a mimics and inhibitor were transfected into HBMSCs, and the transfection efficacy was detected by RT-PCR (Fig. 4d). The cells were assigned to the follow-

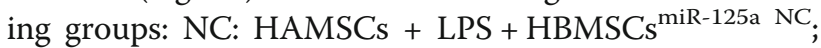
mimics: HAMSCs + LPS + HBMSCs ${ }^{\text {miR-125a mimics; }}$ iNC:
HAMSCs + LPS + HBMSCs ${ }^{\text {miR-125a iNC }}$; inhibitor: HAMS Cs + LPS + HBMSCs ${ }^{\text {miR-125a inhibitor }}$. Moreover, miR125a mimics enhanced ALP staining and activity compared with those in the NC groups, whereas miR-125a inhibitor in HBMSCs inhibited the effect (Fig. 4e). Besides, Alizarin red staining and quantification showed increased matrix mineralization in the miR-125a mimics group compared with the NC groups, whereas miR-125a inhibitor showed the opposite effects (Fig. 4f). RT-PCR and Western blot analysis showed that the mRNA and protein levels of ALP and RUNX2 (early-stage osteogenic markers) and OCN and OSX (late-stage markers) were increased by miR-125a mimics and decreased by miR-125a inhibitor (Fig. 4g, h).

\section{MiR-125a alleviated HAMSC-driven osteogenesis in HBMSCs by targeting APC and activating the $\mathrm{Wnt} / \beta$ - catenin pathway}

APC was selected as the candidate target gene of miR125a using TargetScan software (Fig. 5a). Western blot assay and RT-PCR showed that the mRNA and protein levels of APC were significantly decreased by miR-125a mimics and increased by the miR-125a inhibitor (Fig. 5b, c). The study further explored the mechanisms underlying miR-125a-APC interaction, and a dual-luciferase

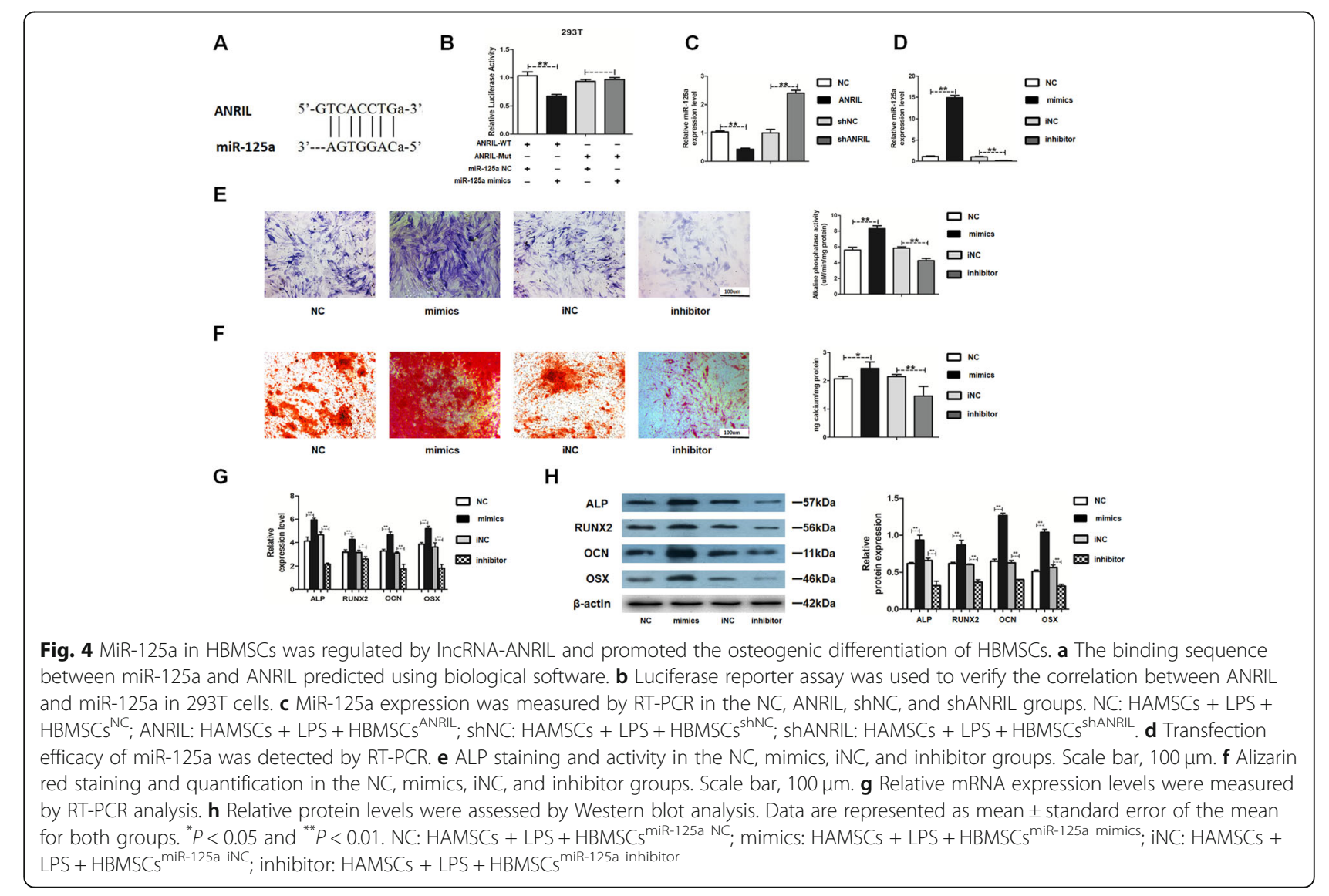


reporter assay was performed. The results suggested that miR-125a mimics reduced the luciferase activity of APC wild-type reporter, while this suppressive effect was rescued by mutation of the putative miR-125a target sites (Fig. 5d).

As APC is a negative regulator in the $\mathrm{Wnt} / \mathrm{\beta}$-catenin pathway [27], APC overexpression was established in HBMSCs and the cells were assigned to the following

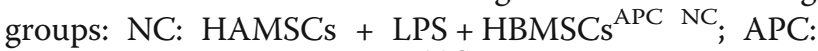
HAMSCs + LPS + HBMSCs ${ }^{\text {APC }}$. Western blot analysis showed that APC overexpression increased the protein level of APC and decreased the protein level of $\beta$ catenin (Fig. 5e). However, the mRNA level of $\beta$-catenin was stable, suggesting that APC overexpression induced only $\beta$-catenin protein degradation without influencing mRNA (Fig. 5f). Immunofluorescence staining further showed a significant decrease in nuclear $\beta$-catenin accumulation when APC was overexpressed (Fig. 5g). Moreover, Western blot analysis and RT-PCR showed that the protein and mRNA levels of ALP and RUNX2 (early-stage osteogenic markers) and OCN and OSX (late-stage markers) were decreased by APC overexpression (Fig. 5h, i).
MiR-125a inhibitor suppressed the APC downregulation and osteogenesis caused by shANRIL

The rescue assays were carried out to fully understand the role of miR-125a and APC in ANRIL-mediated osteogenesis. As shown in Fig. 6a, b, shANRIL and miR-125a inhibitor co-transfection rectified APC suppression compared with that in the shANRIL group. The shANRIL-mediated expression of osteogenic markers was also partly inhibited in co-transfected cells (Fig. 6b, c). shANRIL enhanced ALP staining and activity compared with those in the shNC groups, whereas co-transfection partly inhibited the effect (Fig. 6d). Besides, Alizarin red staining and quantification showed decreased matrix mineralization in the cotransfection group compared with those in the shANRIL groups (Fig. 6e). Taken together, HAMSCs downregulated the expression of lncRNA-ANRIL in LPS-induced HBMSCs, upregulated the miR-125a level, targeted APC transcription, and activated the $\mathrm{Wnt} / \beta$-catenin pathway to induce new bone formation.

\section{Discussion}

The present study demonstrated that lncRNA-ANRIL derived from LPS-pretreated HBMSCs was responsible

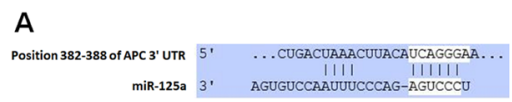

B
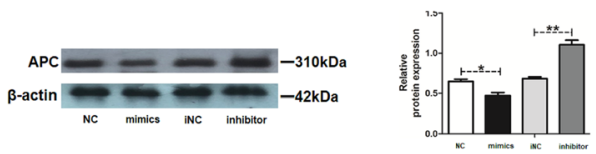

E
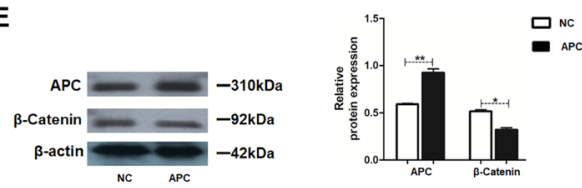

H

G
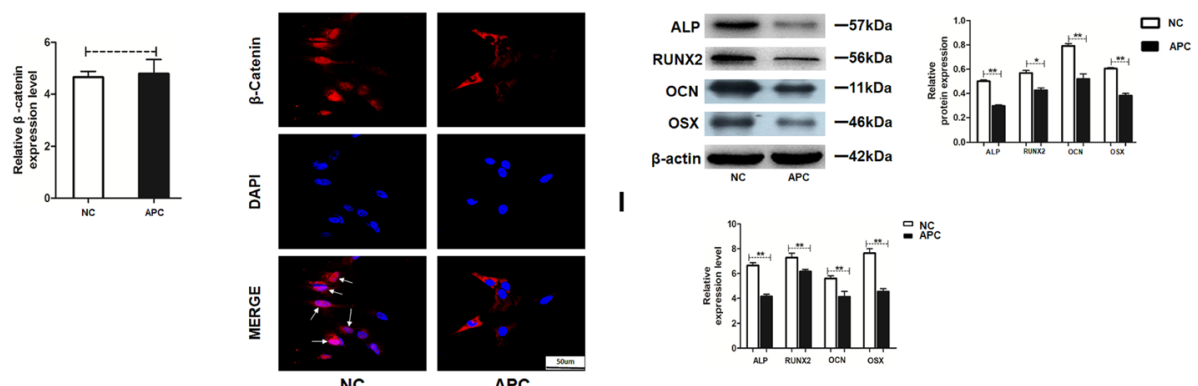

Fig. 5 MiR-125a targeted APC, activated Wnt/ß-catenin pathway, and alleviated osteogenesis. a The potential binding sites between APC and miR-125a predicted using biological software. b APC protein level was assessed by Western blot analysis in the NC, mimics, iNC, and inhibitor groups. c APC mRNA expression was measured by RT-PCR analysis in the NC, mimics, iNC, and inhibitor groups. NC: HAMSCS + LPS +

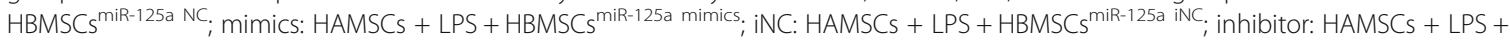

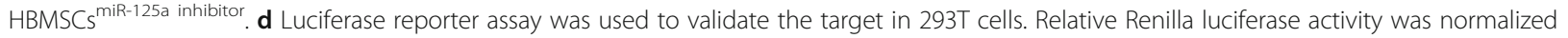
to that of firefly luciferase. e APC protein level was assessed by Western blot analysis in the NC and APC groups. f Relative mRNA expression of $\beta$ catenin was measured by RT-PCR analysis in the NC and APC groups. $\mathbf{g}$ Immunofluorescence staining showed a $\beta$-catenin location in the NC and APC groups. Scale bar, $50 \mu \mathrm{m}$. $\mathbf{h}$ Relative protein levels were assessed by Western blot analysis in the NC and APC groups. i Relative mRNA expression levels were measured by RT-PCR analysis in the NC and APC groups. NC: HAMSCS + LPS + HBMSCSAPC NC; APC: HAMSCS + LPS + HBMSCS $^{\text {APC }}$. Data are represented as mean \pm standard error of the mean for both groups. ${ }^{*} P<0.05$ and ${ }^{* *} P<0.01$ 


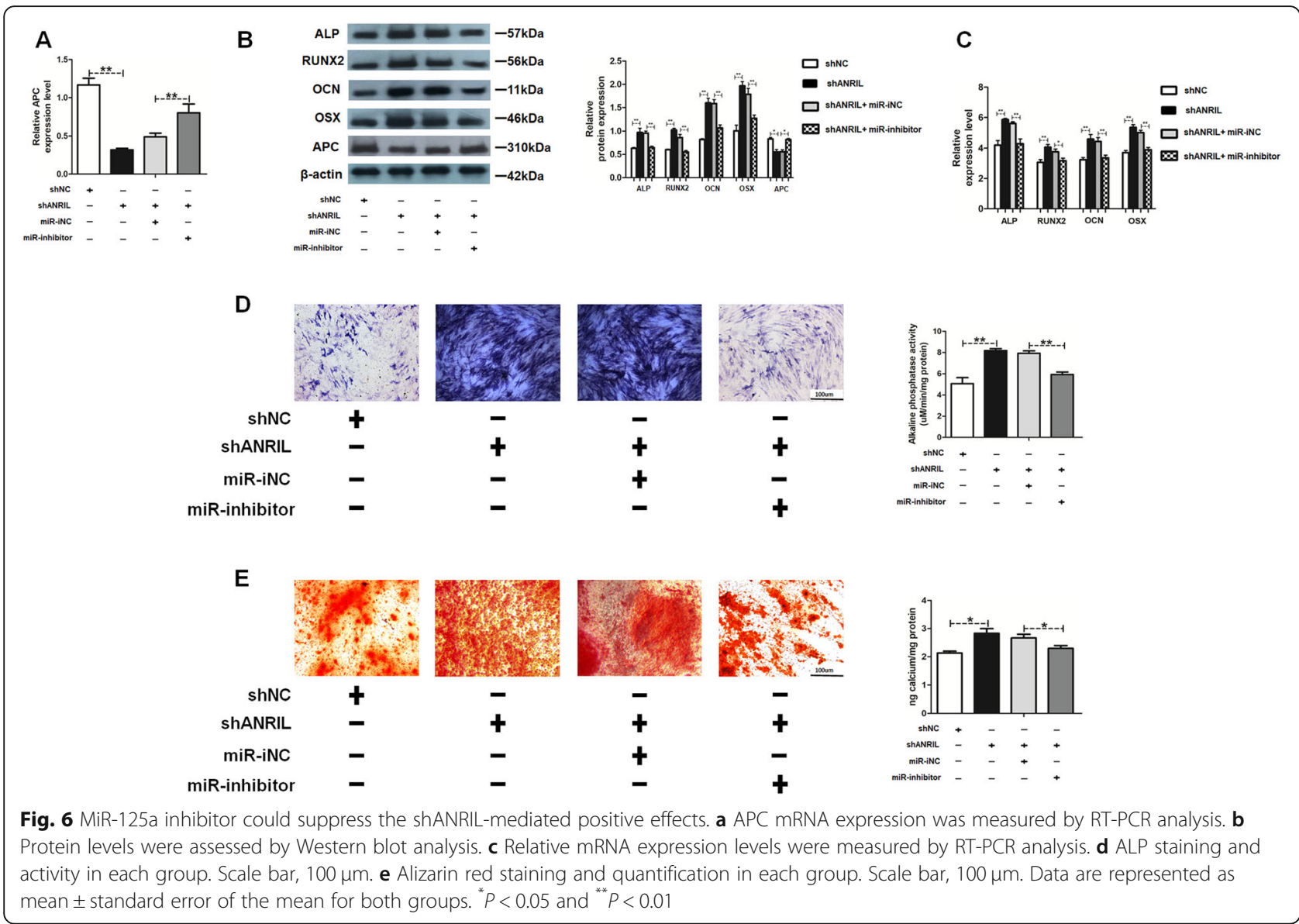

for HAMSC-induced osteogenesis. Briefly, HAMSCs promoted osteogenesis and reduced oxidative stress in LPS-pretreated HBMSCs, and the expression of lncRNA-ANRIL in HBMSCs significantly decreased with HAMSC co-culture. Then, the application of HAMSCs + LPS + HBMSCs ${ }^{\text {shANRIL }}$ into the scaffold material induced bone formation in mandibular defects. Furthermore, miR-125a, acting as a sponge of ANRIL, not only regulated HBMSC function in osteogenesis by targeting APC but also positively regulated osteogenic differentiation via the $\mathrm{Wnt} / \beta$-catenin pathway, suggesting that the axis might be a potentially biological target for treating inflammation-related bone deficiency (Fig. 7). Periodontitis is the most prevalent disease of the alveolar bone, which eventually leads to periodontal tissue and tooth loss. Yet, the current strategies, such as autologous bone and biomaterial transplantation, suffer from limited sources, uncertain outcomes, and high cost $[28,29]$. Tissue regeneration based on stem cells is a promising therapeutic approach. HAMSCs, isolated from the amniotic membrane, is associated with superior immunomodulatory properties and less ethical controversy [4, 30]. A previous study also indicated that decreased inflammation factors and increased growth factors were the potential causes of HAMSC implication in tissue remodeling [30]. At the beginning of the study, it was observed that the osteogenic differentiation and oxidative stress of LPS-induced HBMSCs were significantly alleviated with HAMSCs co-culture, which was consistent with a previous investigation [31]. Importantly, these phenotypic changes in LPS-induced HBMSCs were accompanied by progressively reduced lncRNA-ANRIL expression.

Next, lncRNA-ANRIL was explored in this study. ANRIL, which was formerly regarded as the risk locus associated with coronary heart disease and germline deletion by genome-wide association studies [9, 32], is located in a $42-\mathrm{kb}$ stretch within the chromosomal region 9p21.3. Periodontitis and coronary heart disease share similar pathogenic bacterial strains and environmental and behavioral risk factors [33, 34]. Hence, a recent study suggested a positive correlation between ANRIL and periodontitis across different populations [11]. Furthermore, enhanced ANRIL transcription in bacteriainfected periodontal tissue was upregulated by multiple signaling pathways in response to inflammation [35]. Considering the anti-inflammatory properties of HAMS Cs and decreased ANRIL expression in LPS-induced 


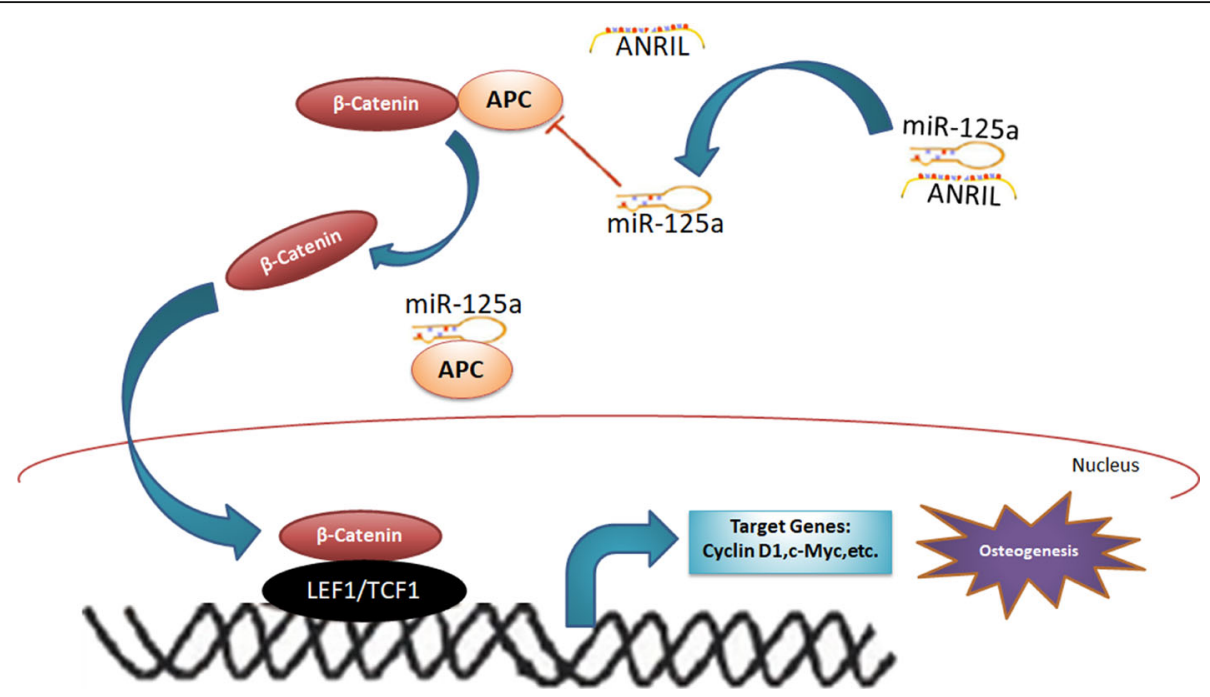

Fig. 7 Schematic working model for IncRNA-ANRIL/miR-125a/APC/ $/$-catenin axis in the regulation of LPS-induced HBMSC osteogenesis

HBMSCs, the present study tested whether HAMSCs performed their function through ANRIL. In this study, HBMSCs $^{\text {ANRIL }}$ led to reversed osteogenesis, while HBMSCs ${ }^{\text {shANRIL }}$ displayed the opposite effects. These data demonstrated that HAMSCs contributed to LPSinduced HBMSC osteoblastogenesis by reducing ANRIL expression.

In the last few years, IncRNAs have been indicated as miRNA sponges to negatively regulate miRNA expression [36]. MiRNAs are involved in multiple cellular processes by influencing gene expression [37]. Thus, it was hypothesized that the effects of ANRIL might be associated with miRNA expression. MiR-125a can participate in cell proliferation, differentiation, and senescence by influencing mRNA stability and translation [38]. The inhibition of ANRIL induces cell apoptosis, which may have a correlation with increased miR-125a expression [15]. Additionally, the association between miR-125a and periodontitis has been investigated in a previous study [39]. The present study demonstrated that miR125a in LPS-induced HBMSCs was directly bound by ANRIL and negatively regulated,while ANRIL was not affected by miR-125a (see Supplementary Material). MiR-125a mimics increased osteogenesis, and miR-125a inhibitor reversed osteogenic differentiation caused by HAMSCs. Collectively, all these data indicated that ANRIL acted as an miR-125a sponge in the underlying mechanism.

The Wnt/ $\beta$-catenin pathway is a classic signal having multiple roles in cellular behaviors [40, 41]. APC, which acts as a negative regulator in the $\mathrm{Wnt} / \beta$-catenin pathway, notably binds to $\beta$-catenin and inhibits its transfer [42]. Targeting APC may emerge as external stimuli, promote the nuclear localization of $\beta$-catenin, and activate the pathway. The present study confirmed that miR-125a bound to the 3 '-UTR of APC, and their expression negatively correlated. Then, the study explored the roles of APC and $\beta$-catenin in the underlying mechanism. The results suggested that APC overexpression intuitively decreased the nuclear localization of $\beta$ catenin and contributed to osteoblast repression in HBMSCs. Moreover, the rescue assays showed that shANRIL and miR-125a inhibitor co-transfection rectified APC suppression and inhibited osteogenic differentiation. Thus, it is believed that the interaction between $\mathrm{APC}$ and $\beta$-catenin serves as a downstream regulator of miR-125a in ANRIL-induced osteogenesis.

\section{Conclusions}

In conclusion, the findings of the present study supported the hypothesis that HAMSCs promoted the osteogenic differentiation of LPS-induced HBMSCs via the lncRNA-ANRIL/miR-125a/APC/ $\beta$-catenin axis. Based on these results, a viable therapeutic target and paramount approach may be proposed for the therapy of inflammatory bone-destructive processes.

\section{Supplementary Information}

The online version contains supplementary material available at https://doi. org/10.1186/s13287-020-02105-8.

Additional file 1. Other relevant datasets

\section{Abbreviations}

ALP: Alkaline phosphatase; ANRIL: Antisense noncoding RNA in the INK4 locus; APC: Adenomatous polyposis coli; BV/TV: Bone volume/total volume; DCF: 2',7'-Dichlorofluorescein; GAPDH: Glyceraldehyde-3-phosphate

dehydrogenase; HAMSCs: Human amnion-derived mesenchymal stem cells; HBMSCs: Human bone marrow mesenchymal stem cells; H\&E: Hematoxylin and eosin; IncRNAs: Long noncoding RNAs; LPS: Lipopolysaccharide; micro- 
CT: Micro-computed tomography; miR-125a: MicroRNA-125a; miRNAs: MicroRNAs; NcRNAs: Noncoding RNAs; OCN: Osteocalcin; OSX: Osterix; ROS: Reactive oxygen species; RT-PCR: Quantitative real-time reverse transcription-polymerase chain reaction; SOD: Superoxide dismutase; SSRO: Sagittal split ramus osteotomy

\section{Acknowledgements}

Not applicable.

\section{Authors' contributions}

Yuli Wang performed the experiments. Fengyi Lv and Lintong Huang supervised the study. Hengwei Zhang, Bing Li, and Weina Zhou interpreted the data and wrote the original draft. Xuan Li performed some experiments and data analysis. Yifei Du designed the experiments. Yongchu Pan and Ruixia Wang wrote the manuscript. The authors have read and approved the final version of the manuscript.

\section{Funding}

This study was supported by grants from the Priority Academic Program Development of Jiangsu Higher Education Institutions (PAPD, No. 2018-87); the Jiangsu Provincial Commission of Health and Family Planning, Nanjing, China (No. BJ18035); the National Natural Science Foundation of China (No. 81800936); the Natural Science Foundation of Jiangsu Province (No. BK20180668, BK20190648); the Southeast University-Nanjing Medical University Cooperative Research Project (No. 2242018K3DN17); the China Postdoctoral Science Foundation Funded Project (No. 2018 M64050 and 2019T120445); the Jiangsu Postdoctoral Science Foundation Funded Project (No. 2018K251C); the Jiangsu Province Undergraduate Innovation and Entrepreneurship Training Program (No. 201910312004Z); and the Open Research Fund of Jiangsu Key Laboratory of Oral Diseases (No. JSKLOD-KF-1903).

\section{Availability of data and materials}

All other relevant datasets have been uploaded as part of additional files.

\section{Ethics approval and consent to participate}

All human stem cells involved in the present study followed the International Society for Stem Cell Research "Guidelines for the Conduct of Human Embryonic Stem Cell Research." The study was approved by the ethics and research committee of Nanjing Medical University (Permit Number: 2018-190). Informed consent was obtained from all the participants.

\section{Consent for publication}

Not applicable.

\section{Competing interests}

The authors declare no conflicts of interest.

\section{Author details}

${ }^{1}$ Jiangsu Key Laboratory of Oral Diseases, Nanjing Medical University, Nanjing, China. ${ }^{2}$ Department of Oral and Maxillofacial Surgery, Affiliated Hospital of Stomatology, Nanjing Medical University, Nanjing, China. ${ }^{3}$ Department of Pathology and Laboratory Medicine and Center for Musculoskeletal Research, University of Rochester Medical Center, Rochester, New York, USA ${ }^{4}$ Department of Temporomandibular Joint, Affiliated Hospital of Stomatology, Nanjing Medical University, Nanjing, China. ${ }^{5}$ Department of Orthodontics, Affiliated Hospital of Stomatology, Nanjing Medical University, Nanjing, China. ${ }^{6}$ Department of Dental Implant, Affiliated Hospital of Stomatology, Nanjing Medical University, Nanjing, China.

Received: 13 July 2020 Accepted: 14 December 2020

Published online: 07 January 2021

\section{References}

1. Hajishengallis G. Immunomicrobial pathogenesis of periodontitis: keystones, pathobionts, and host response. Trends Immunol. 2014;35(1):3-11.

2. How KY, Song KP, Chan KG. Porphyromonas gingivalis: an overview of periodontopathic pathogen below the gum line. Front Microbiol. 2016;7:53.

3. Macchiarini $P$, Jungebluth $P$, Go T, Asnaghi MA, Rees LE, Cogan TA, et al Clinical transplantation of a tissue-engineered airway. Lancet. 2008; 372(9655):2023-30.
4. Lobo SE, Leonel LC, Miranda CM, Coelho TM, Ferreira GA, Mess A, et al. The placenta as an organ and a source of stem cells and extracellular matrix: a review. Cells Tissues Organs. 2016;201(4):239-52.

5. Wang Y, Wu H, Shen M, Ding S, Miao J, Chen N. Role of human amnionderived mesenchymal stem cells in promoting osteogenic differentiation by influencing p38 MAPK signaling in lipopolysaccharide -induced human bone marrow mesenchymal stem cells. Exp Cell Res. 2017;350(1):41-9.

6. Schmitz SU, Grote P, Herrmann BG. Mechanisms of long noncoding RNA function in development and disease. Cell Mol Life Sci. 2016;73(13):2491-509.

7. Fatica A, Bozzoni I. Long non-coding RNAs: new players in cell differentiation and development. Nat Rev Genet. 2014;15(1):7-21.

8. Geisler S, Coller J. RNA in unexpected places: long non-coding RNA functions in diverse cellular contexts. Nat Rev Mol Cell Biol. 2013;14(11):699-712.

9. Pasmant E, Laurendeau I, Heron D, Vidaud M, Vidaud D, Bieche I. Characterization of a germ-line deletion, including the entire INK4/ARF locus, in a melanoma-neural system tumor family: identification of ANRIL, an antisense noncoding RNA whose expression coclusters with ARF. Cancer Res. 2007;67(8):3963-9.

10. Bochenek G, Hasler R, El Mokhtari NE, Konig IR, Loos BG, Jepsen S, et al. The large non-coding RNA ANRIL, which is associated with atherosclerosis, periodontitis and several forms of cancer, regulates ADIPOR1, VAMP3 and C11ORF10. Hum Mol Genet. 2013;22(22):4516-27.

11. Schaefer AS, Bochenek G, Manke T, Nothnagel M, Graetz C, Thien A, et al. Validation of reported genetic risk factors for periodontitis in a large-scale replication study. J Clin Periodontol. 2013:40(6):563-72.

12. Schaefer AS, Richter GM, Dommisch $H$, Reinartz $M$, Nothnagel M, Noack $B$, et al. CDKN2BAS is associated with periodontitis in different European populations and is activated by bacterial infection. J Med Genet. 2011;48(1): $38-47$.

13. Lou G, Dong X, Xia C, Ye B, Yan Q, Wu S, et al. Direct targeting spermassociated antigen 9 by miR-141 influences hepatocellular carcinoma cell growth and metastasis via JNK pathway. J Exp Clin Cancer Res. 2016:35:14.

14. Li R, Yin F, Guo YY, Zhao KC, Ruan Q, Qi YM. Knockdown of ANRIL aggravates $\mathrm{H} 2 \mathrm{O} 2$-induced injury in PC-12 cells by targeting microRNA-125a. Biomed Pharmacother. 2017;92:952-61.

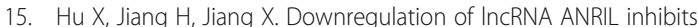
proliferation, induces apoptosis, and enhances radiosensitivity in nasopharyngeal carcinoma cells through regulating miR-125a. Cancer Biol Ther. 2017;18(5):331-8.

16. Zhang D, Jiang M, Miao D. Transplanted human amniotic membranederived mesenchymal stem cells ameliorate carbon tetrachloride-induced liver cirrhosis in mouse. PLoS One. 2011;6(2):e16789.

17. Akintoye SO, Lam T, Shi S, Brahim J, Collins MT, Robey PG. Skeletal sitespecific characterization of orofacial and iliac crest human bone marrow stromal cells in same individuals. Bone. 2006:38(6):758-68.

18. Dong $X$, Jin Z, Chen $Y, X u H, M a ~ C$, Hong $X$, et al. Knockdown of long noncoding RNA ANRIL inhibits proliferation, migration, and invasion but promotes apoptosis of human glioma cells by upregulation of miR-34a. J Cell Biochem. 2018:119(3):2708-18.

19. Zhao Q, Shao L, Hu X, Wu G, Du J, Xia J, et al. Lipoxin a4 preconditioning and postconditioning protect myocardial ischemia/reperfusion injury in rats. Mediat Inflamm. 2013;2013:231351

20. Wang J, Miao J, Meng $X$, Chen N, Wang Y. Expression of long noncoding RNAs in human bone marrow mesenchymal stem cells cocultured with human amnionderived mesenchymal stem cells. Mol Med Rep. 2017;16(5): 6683-9.

21. Ge W, Shi L, Zhou Y, Liu Y, Ma GE, Jiang Y, et al. Inhibition of osteogenic differentiation of human adipose-derived stromal cells by retinoblastoma binding protein 2 repression of RUNX2-activated transcription. Stem Cells. 2011:29(7):1112-25.

22. Ge W, Liu Y, Chen T, Zhang $X, L v L$, Jin $C$, et al. The epigenetic promotion of osteogenic differentiation of human adipose-derived stem cells by the genetic and chemical blockade of histone demethylase LSD1. Biomaterials. 2014:35(23):6015-25

23. Ma X, Bian $Y$, Yuan $H$, Chen N, Pan $Y$, Zhou W, et al. Human amnion-derived mesenchymal stem cells promote osteogenic differentiation of human bone marrow mesenchymal stem cells via H19/miR-675/APC axis. Aging (Albany NY). 2020;12(11):10527-43.

24. Jia LF, Wei SB, Gan YH, Guo Y, Gong K, Mitchelson K, et al. Expression, regulation and roles of miR-26a and MEG3 in tongue squamous cell carcinoma. Int J Cancer. 2014;135(10):2282-93. 
25. Xu R, Shen X, Si Y, Fu Y, Zhu W, Xiao T, et al. MicroRNA-31a-5p from aging BMSCs links bone formation and resorption in the aged bone marrow microenvironment. Aging Cell. 2018;17(4):e12794.

26. Zhou H, Rigoutsos I. MiR-103a-3p targets the $5^{\prime}$ UTR of GPRC5A in pancreatic cells. RNA. 2014;20(9):1431-9.

27. Ni L, Kuang Z, Gong Z, Xue D, Zheng Q. Dihydroartemisinin promotes the osteogenesis of human mesenchymal stem cells via the ERK and Wnt/betacatenin signaling pathways. Biomed Res Int. 2019;2019:3456719.

28. Leng $Q$, Chen L, Lv Y. RNA-based scaffolds for bone regeneration: application and mechanisms of mRNA, miRNA and siRNA. Theranostics. 2020;10(7):3190-205

29. Tang D, Tare RS, Yang LY, Williams DF, Ou KL, Oreffo RO. Biofabrication of bone tissue: approaches, challenges and translation for bone regeneration. Biomaterials. 2016;83:363-82.

30. Silini AR, Magatti M, Cargnoni A, Parolini O. Is immune modulation the mechanism underlying the beneficial effects of amniotic cells and their derivatives in regenerative medicine? Cell Transplant. 2017;26(4):531-9.

31. Wang Y, Ma J, Du Y, Miao J, Chen N. Human amnion-derived mesenchymal stem cells protect human bone marrow mesenchymal stem cells against oxidative stress-mediated dysfunction via ERK1/2 MAPK signaling. Mol Cells. 2016;39(3):186-94.

32. Coronary Artery Disease C, Samani NJ, Deloukas P, Erdmann J, Hengstenberg C, Kuulasmaa K, et al. Large scale association analysis of novel genetic loci for coronary artery disease. Arterioscler Thromb Vasc Biol. 2009;29(5):774-80.

33. Chiu B. Multiple infections in carotid atherosclerotic plaques. Am Heart 1999;138(5 Pt 2):S534-6.

34. Tonetti MS, D'Aiuto F, Nibali L, Donald A, Storry C, Parkar M, et al. Treatment of periodontitis and endothelial function. N Engl J Med. 2007;356(9):911-20.

35. Harismendy O, Notani D, Song X, Rahim NG, Tanasa B, Heintzman N, et al. 9p21 DNA variants associated with coronary artery disease impair interferon-gamma signalling response. Nature. 2011:470(7333):264-8.

36. Militello G, Weirick T, John D, Doring C, Dimmeler S, Uchida S. Screening and validation of IncRNAs and circRNAs as miRNA sponges. Brief Bioinform. 2017;18(5):780-8.

37. Davis HM, Pacheco-Costa R, Atkinson EG, Brun LR, Gortazar AR, Harris J, et al. Disruption of the CX43/miR21 pathway leads to osteocyte apoptosis and increased osteoclastogenesis with aging. Aging Cell. 2017;16(3):551-63.

38. Potenza N, Russo A. Biogenesis, evolution and functional targets of microRNA-125a. Mol Gen Genomics. 2013;288(9):381-9.

39. Venugopal P, Lavu V, Rao SR, Venkatesan V. Association of microRNA-125a and microRNA-499a polymorphisms in chronic periodontitis in a sample south Indian population: a hospital-based genetic association study. Gene. 2017:631:10-5

40. Zhou Y, Li J, Zhou K, Liao X, Zhou X, Shen K. The methylation of Notch1 promoter mediates the osteogenesis differentiation in human aortic valve interstitial cells through Wnt/beta-catenin signaling. J Cell Physiol. 2019; 234(11):20366-76

41. Wang Y, Zhang X, Shao J, Liu H, Liu X, Luo E. Adiponectin regulates BMSC osteogenic differentiation and osteogenesis through the Wnt/beta-catenin pathway. Sci Rep. 2017;7(1):3652.

42. Qu Y, Gharbi N, Yuan X, Olsen JR, Blicher P, Dalhus B, et al. Axitinib blocks Wnt/beta-catenin signaling and directs asymmetric cell division in cancer. Proc Natl Acad Sci U S A. 2016;113(33):9339-44.

\section{Publisher's Note}

Springer Nature remains neutral with regard to jurisdictional claims in published maps and institutional affiliations.

Ready to submit your research? Choose BMC and benefit from:

- fast, convenient online submission

- thorough peer review by experienced researchers in your field

- rapid publication on acceptance

- support for research data, including large and complex data types

- gold Open Access which fosters wider collaboration and increased citations

- maximum visibility for your research: over $100 \mathrm{M}$ website views per year

At BMC, research is always in progress.

Learn more biomedcentral.com/submissions 